\title{
The Study of a Super Low Altitude Satellite
}

\author{
By Atsushi Noda, Masanori Homma and Masayoshi Utashima \\ Japan Aerospace Exploration Agency (JAXA), Tsukuba, Japan
}

(Received May 7th, 2008)

\begin{abstract}
This paper reports the result of a study for super low altitude satellite. The altitude of this satellite's orbit is lower than ever. The altitude of a conventional earth observing satellite is generally around from $600 \mathrm{~km}$ to $900 \mathrm{~km}$. The lowest altitude of earth observing satellite launched in Japan was 350km; the Tropical Rainfall Measuring Mission (TRMM). By comparison, the satellite reported in this paper is much lower than that and it is planned to orbit below 200km. Furthermore, the duration of the flight planned is more than two years. Any satellite in the world has not achieved to keep such a low altitude that long term. The satellite in such a low orbit drops quickly because of the strong air drag. Our satellite will cancel the air drag effect by ion engine thrust. To realize this idea, a drag-free system will be applied. This usually leads a complicated and expensive satellite system. We, however, succeeded in finding a robust control law for a simple system even under the unpredictable change of air drag. When the altitude of the satellite is lowered successfully, the spatial resolution of an optical sensor can be highly improved. If a SAR is equipped with the satellite, it enables the drastic reduction of electric power consumption and the fabulous spatial resolution improvement at the same time.
\end{abstract}

Key Words: Ion engine, Satellite, Orbit

\section{Introduction}

This paper describes the result of a study for a super low altitude satellite. The altitude of this satellite's orbit is lower than ever. The altitude of a conventional earth observing satellite is generally around from $600 \mathrm{~km}$ to $900 \mathrm{~km}$. The lowest altitude of earth observing satellite launched in Japan was 350km; the Tropical Rainfall Measuring Mission (TRMM). Comparing with that, the satellite reported in this paper is planned to orbit below $200 \mathrm{~km}$ which is kept by the ion engine's thrust.

Primary sensor candidates for this super low altitude satellite are conceived to be as follows:

(1)Optical Camera

(2)Synthetic Aperture Radar (SAR)

(3)Light Detection and Ranging (LIDAR).

\subsection{Optical camera}

When an optical camera is installed in the super low altitude satellite, the merit we obtain is in the spatial resolution. The spatial resolution of optical cameras is proportional to the altitude. Therefore, even if the same camera is used, the spatial resolution is improved by 4 , when the altitude is lowered to $1 / 4$ of the conventional satellite altitude. On the contrary, a low cost camera with a smaller aperture can be used, when the improvement of the spatial resolution is not required.

There is, however, drawbacks from the lower altitude such as the narrow swath width, the lower signal to noise ratio, and the larger data volume.

\subsection{Synthetic aperture radar (SAR)}

The electric power required by SAR is proportional to the third power of the altitude. When the $1 / 4$ altitude of the conventional one is used, the required electric power is lowered to $1 / 64$. Approaches which make use of the merits of power saving are as follows:

(1)to realize the conventional spatial resolution by the lower electric power

(2)to realize the better spatial resolution by the conventional electric power

(3)to realize the $\mathrm{Ku}$ band SAR with about $50 \mathrm{~cm}$ spatial resolution

\subsection{Light detection and ranging (LIDAR)}

LIDAR requires the laser power which is proportional to the second power of the altitude. One of the difficulties for the onboard LIDAR development is from high power laser transmitter. The design of the heat exhaust system and the prevention of the burnout are important points. If the super low altitude satellite, however, is realized a practical LIDAR can be developed because of the decreased laser power requirement.

\section{Altitude of Super Low Altitude Satellite}

\subsection{Aerodynamic study}

By the study of the air drag for the super low altitude satellite, it is found that the satellite shape such as the streamline or the wedge cannot be effective to decrease air drag, because of the high speed and rarefied air density. Regardless of the shape of the satellite, the drag coefficient (CD) is almost 2.0. The air drag (F) can be determined simply by the front projection area (S) as follows:

$$
F=\frac{1}{2} \rho V^{2} S C_{D}
$$

Our aerodynamic study shows that merits from the lift and the contributions of the tails to the attitude stability are not recognized.

\subsection{Air density}


We evaluated the air density by the ICAO standard atmosphere. Fig. 1 shows the relation between the altitude and the air density. This model almost corresponds to the atmosphere at the solar maximum period. Therefore, the study in this paper corresponds to the worst case.

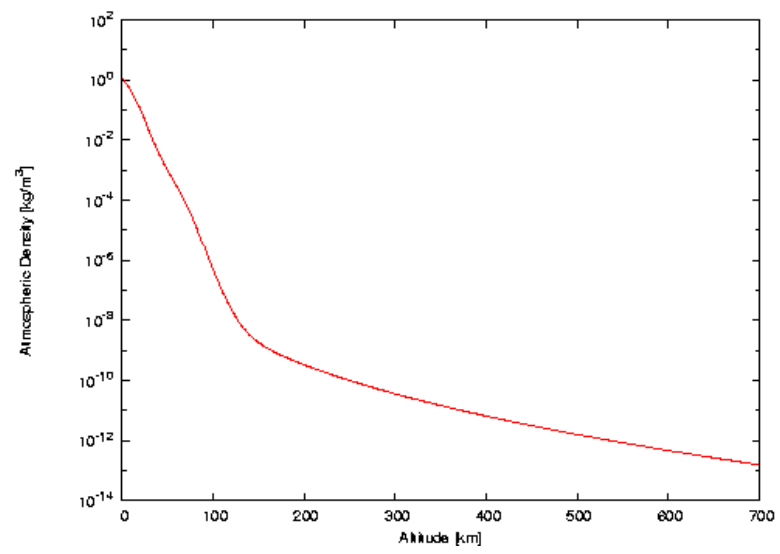

Fig.1. Altitude and air density relationship

\subsection{Ion engine}

In order to maintain the satellite's altitude, ion engines have to keep on thrusting. Since ion engines have high specific impulses, they require only small amount of fuel. Therefore, our super low altitude satellite can have enough amount of fuel on board which enables the flight period more than two years. On the other hand, since ion engines require large amount of the electric power, the solar battery with large area is required.

The maximum problem is that ion engines cancel the air drag of the large area solar battery and the satellite body. The air drag depends on the satellite altitude. The analysis of air drag and altitude is shown in the next paragraph.

\subsection{Altitude vs. air drag, power consumption, and fuel amount}

Table 2-1 shows the required ion engine's thrust (=drag), electric power, and fuel amount for maintaining at each altitude.

Table 2-1. Altitude vs. drag, power, fuel

\begin{tabular}{|c|l|l|c|l|}
\hline $\begin{array}{c}\text { alt. } \\
{[\mathrm{km}]}\end{array}$ & $\begin{array}{c}\text { air dens. } \\
{\left[\mathrm{kg} / \mathrm{m}^{3}\right]}\end{array}$ & $\begin{array}{c}\text { drag } \\
{\left[\mathrm{N} / \mathrm{m}^{2}\right]}\end{array}$ & $\begin{array}{c}\text { power } \\
{\left[\mathrm{W} / \mathrm{m}^{2}\right]}\end{array}$ & $\begin{array}{c}\text { fuel* } \\
{[\mathrm{kg} / \mathrm{y}]}\end{array}$ \\
\hline 160. & $1.16 \mathrm{e}-9$ & 0.0707 & 2121. & 75.8 \\
\hline 170. & $8.04 \mathrm{e}-10$ & 0.0489 & 1468. & 52.5 \\
\hline 180. & $5.86 \mathrm{e}-10$ & 0.0356 & 1069. & 38.2 \\
\hline 190. & $4.35 \mathrm{e}-10$ & 0.0264 & 792. & 28.3 \\
\hline 200. & $3.32 \mathrm{e}-10$ & 0.0201 & 604. & 21.6 \\
\hline 210. & $2.56 \mathrm{e}-10$ & 0.0155 & 465. & 16.6 \\
\hline
\end{tabular}

${ }^{*)}$ For $1 \mathrm{~m}^{2}$ of the front projection area

The front projection area of the satellite determines the ion engine's thrust, fuel amount, electric power, and area of the solar battery. This is quite different from the conventional satellites in which the satellite's mass is a rule factor.

From these studies, we found that the super low altitude satellite can fly at the altitude of $180 \mathrm{~km}$ for more than 2 years.

The best configuration of the super low altitude satellite is with small front projection area and large side area for solar battery. See Fig.2 as illustrated below.

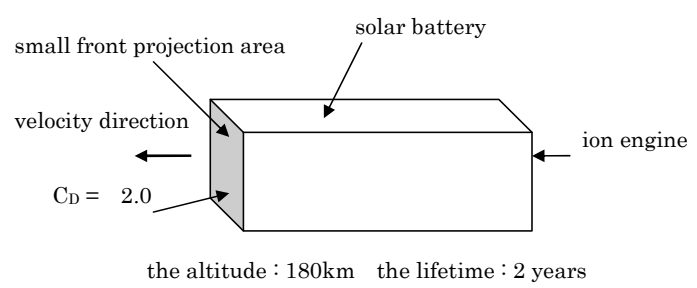

Fig.2. Basic cofiguretion of super low altitude satellite.

\subsection{Atomic oxygen}

The atmosphere around the low altitude of about $180 \mathrm{~km}$ has the different composition from that of the ground level. The most significant difference is that the oxygen atoms don't form molecules but stay as the atoms. The atomic oxygen will deteriorate the material of the satellite. Countermeasure is left for a future task.

\section{Orbital Analysis}

The satellite's altitude in this paper is kept around $180 \mathrm{~km}$ by the ion engine's thrust which cancels the air drag. The air density changes greatly even for a revolution. The drag-free system, which can accurately cancel the perturbations due to non-conservative forces, enables the satellite to be affected only by the geopotential and to fly the frozen orbit ${ }^{1)}$, which has the constant mean eccentricity vector and has been used for many earth observation satellites. Use of the drag-free system, however, makes the satellite system significantly complicated and increases the satellite's cost.

Therefore, we studied whether an ion engine with a simplified throttling function can realize a constant mean eccentricity vector. Then, we have obtained the result that the ion engine thrust, which is constant for one revolution, can keep the mean eccentricity vector as well as the altitude almost constant under the geopotential (J2, J3 terms) and the air drag. We will call the kept orbit as "new frozen orbit."

The concept of the "new frozen orbit" is described in section 3.1, and the results of the constant thrust simulation are shown in section 3.2. Several simulations for maintaining orbits by the simplest on-off thrust control of the ion engine are shown in section 3.3 .

\subsection{Concept of "new frozen orbit"}

The concept of the "new frozen orbit," which can be realized by the constant thrust under the J2, J3 terms and the air drag, is stated.

\section{Velocity field in mean eccentricity vector plane}

The velocity field in the mean eccentricity vector plane is to be explained first. The eccentricity vector plane consists of both $e \cos \omega$ as x-axis and $e \sin \omega$ as y-axis (see Fig.3). $e$ is the eccentricity, and $\omega$ is the argument of perigee. $e$ and $\omega$ in the mean eccentricity vector plane are mean elements which are obtained by removing the short period variations due to perturbations. The black velocity field in Fig.3 is derived by the J2, J3 terms. The point A is the current frozen eccentricity 
vector. The black velocity field has the magnitude proportional to the distance from the point $\mathrm{A}$. On the other hand, the red velocity field is derived by the air drag. The existence of the eccentricity causes both the large air drag around the perigee and the small one around the apogee. That difference of the air drag makes the orbit approach to a circular one. In other words, the mean eccentricity vector has the velocity field toward the origin. The larger the mean eccentricity is, the larger the velocity is.

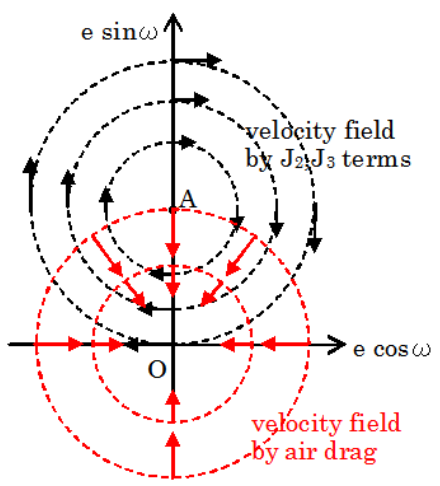

Fig.3. Velocity field of mean eccentricity vector

Possible region of "new frozen eccentricity vector"

The mean eccentricity vector of the "new frozen orbit" can exist in the region where the summed velocity can be zero in Fig.3. The region where the directions of black and red arrows are reverse is the possible region of the "new frozen eccentricity vector.” The blue half circle in Fig. 4 is that region. If the black and red arrows in Fig. 4 have the same magnitude, the point $B$ in Fig.4 is the "new frozen eccentricity vector."

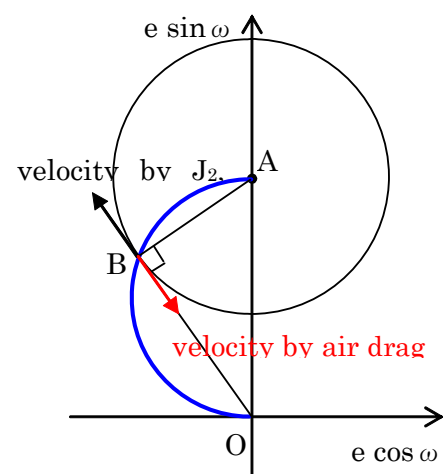

Fig.4. Possible region of "new frozen eccentricity vector"

\section{Change of magnitude of two arrows}

Next, we study the change of the magnitude of two arrows in Fig.4 along the blue arc. The result is shown in Fig.5. When the velocity magnitude by the air drag at the point $\mathrm{A}$ in the solar maximum period is set to be 1.0 , the velocity magnitude by the $\mathrm{J} 2$ and $\mathrm{J} 3$ terms at the origin is about 0.7. When a point moves from the point $\mathrm{A}$ to the origin, the velocity magnitude by the air drag decreases monotonously from 1.0 to 0.0 , and the velocity magnitude by the $\mathrm{J} 2$ and $\mathrm{J} 3$ terms increases monotonously from 0.0 to 0.7 . Therefore, it is clear that the intersection does exist between the point A and the origin. That intersection is the "new frozen eccentricity vector." In the solar minimum period, the velocity magnitude by the air drag at the point $\mathrm{A}$ is about 0.4 . When the solar activity weakens, the "new frozen eccentricity vector" approaches to the point A. When the solar activity strengthens, it approaches to the zero eccentricity.

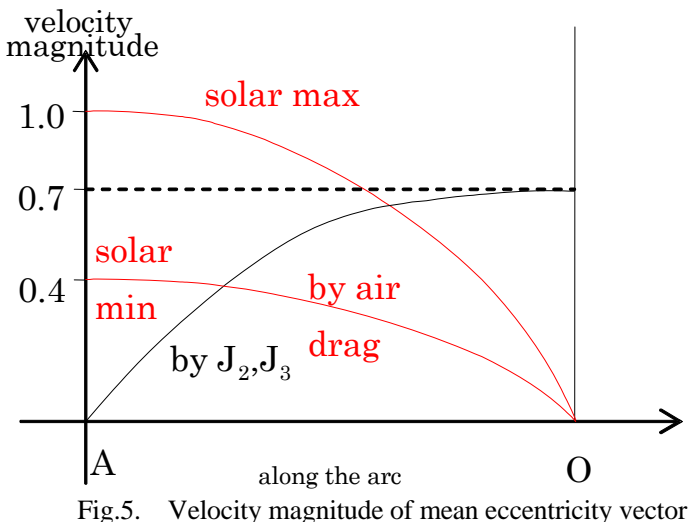

\section{Stability of "new frozen eccentricity vector"}

The current frozen eccentricity vector does not have any restitutive forces, so its stability is neutral. The "new frozen eccentricity vector," however, has a positive stability, that is, when it moves from the balanced position, restitutive forces put it back toward the position. Fig.6 shows this. Black arrows in Fig.6 are the velocity vectors around the point $B$ which is the "new frozen eccentricity vector." From Fig.6, it turns out that the velocity field converges to the point $\mathrm{B}$.

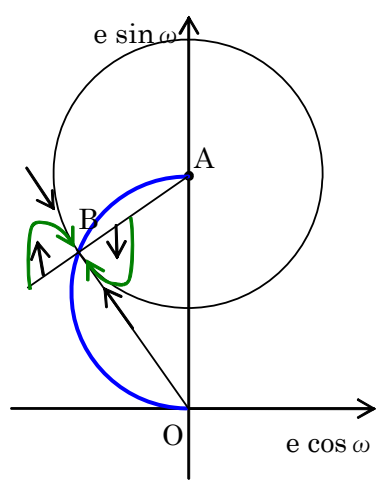

Fig.6. Stability of "new frozen eccentricity vector"

\section{Instability of altitude control}

While the mean eccentricity vector is stable under the constant thrust, the altitude control has instability. If the altitude increases slightly, the air density decreases and the altitude increases further. For the case of the decrement of the altitude, the altitude control is instable as well. Therefore, the altitude must be controlled properly with continuous measurement of the altitude change.

\subsection{Dozens of days' simulation with constant thrust}

In this section, we show by the simulations using the STK/Astrogator that the "new frozen orbit" surely exists and it can be applied to the real missions. We state the conditions of the simulations in section 3.2.1, and show the results of the simulations for 5 cases in section 3.2.2.

\subsubsection{Conditions of simulations}

The conditions of the simulations which are used in the following sections are described below.

Initial osculating orbital elements (input to Astrogator) 
Epoch: January the 1st of 2006, 6h UT

$\mathrm{a}=6567.952 \mathrm{~km}$

e $=0.001246$ (eccentricity of frozen orbit under J2, J3)

$\mathrm{i}=96.3 \mathrm{deg}$

$$
\Omega=\left\{\begin{array}{cc}
10.678 \operatorname{deg} & \left(\mathrm{DLT}^{*}=6 \mathrm{~h}\right) \\
78.178 \mathrm{deg} & (\mathrm{DLT}=10 \mathrm{~h} 30 \mathrm{~m})
\end{array}\right.
$$

$\omega=65.11 \mathrm{deg}$

(argument of perigee of frozen orbit under J2, J3)

$\mathrm{f}$ (true anomaly) $=-65.11 \mathrm{deg}$

This osculating elements were calculated by adding the short period variations of a and eccentricity vector to the mean elements whose altitude at the equator is about $180 \mathrm{~km}$.

Conditions of orbit generation in Astrogator

(1) geopotential: J2, J3 terms

(2) air density model: Jacchia-Roberts solar maximum period: solar flux $=270$ solar minimum period: solar flux $=60$

(3) satellite parameters mass: $500 \mathrm{~kg}$ front projection area (S): $1 \mathrm{~m} 2$ drag coefficient (CD): 2.0

(4) specific impulse of ion engine: $3000 \mathrm{sec}$

\subsubsection{Simulation results}

In this section, we show the results of the simulation for the "new frozen orbit." For easiness, first we obtained the ion engine's thrust which made the satellite's altitude almost constant during dozens of days for each case, then we simulated the change of the mean eccentricity vector by using the thrust obtained above.

The altitude change for both the solar maximum period and the DLT of $6 \mathrm{~h}$ is depicted in Fig.7 as the representative of each case. The thrust of $29.78963 \mathrm{mN}$ was used in this case. Simulations of the "new frozen orbit" for about 40 days are possible.

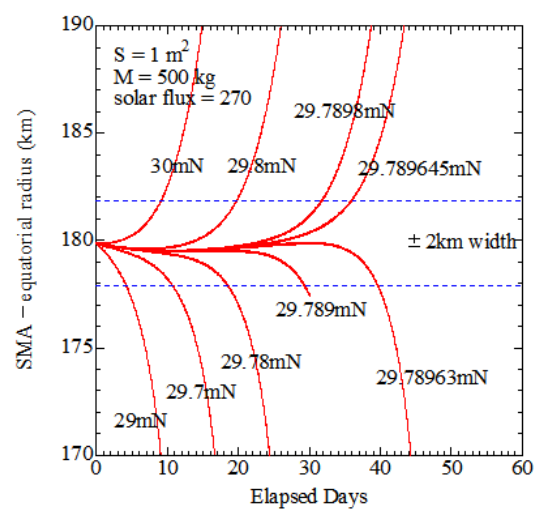

Fig.7. Altitude change (DLT=6h)

The changes of the mean eccentricity vector in the five cases are shown in Fig.8. The point A in Fig.8 is the initial mean eccentricity vector for the four cases. One case was simulated from the point a in Fig.8. The mean eccentricity vector converges to the same point even if a different initial eccentricity vector is used. Any mean eccentricity vectors in Fig.8 significantly change their directions at about 30days. The altitudes after about 30days are not kept constant and go far away from $180 \mathrm{~km}$.

In any case, the "new frozen eccentricity vector" is reached in about 30days. The "new frozen eccentricity vectors" for the DLT of $10 \mathrm{~h} 30 \mathrm{~m}$ are shifted to the positive direction of the $\mathrm{x}$-axis compared to the case for the DLT of $6 \mathrm{~h}$.

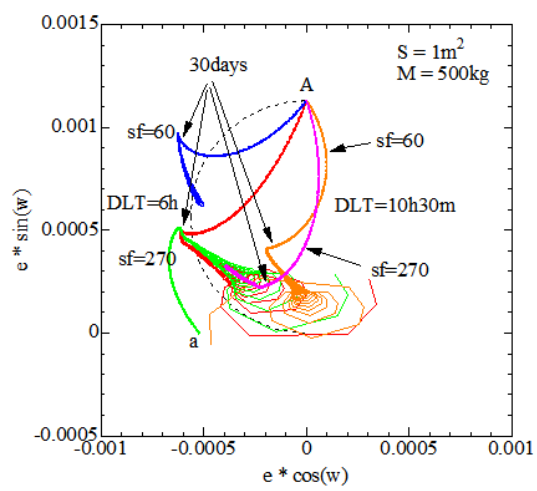

Fig.8. Changes of mean eccentricity vector

\subsection{Simulations of orbit maintenance with on-off control}

Actually, the air density fluctuates from day to day. Therefore, for the maintenance of the orbit, we must know the mean altitude for some interval and adjust the thrust of the ion engine. One orbital period is considered to be suitable as the "some interval." Because the accurate mean orbital elements can be easily obtained by averaging the osculating orbital elements, which are derived from the GPS receiver every moment, for one orbital period.

Though it is desired that the ion engine has the fine throttling function for this mission, the ion engine supposed to be used for the demonstration satellite does not have any throttling function. Therefore, we have studied the orbital maintenance by the on-off control of the ion engine with the fixed thrust. The minimum unit for both the on and off intervals was set to be one orbital period in order to obtain the accurate mean orbital elements.

On the other hand, the frequent switching of the discharge power supply for the ion engine will cause a problem of the short lifetime. Therefore, we are planning that the discharge power supply is kept on and the switching of the thrust is made by the switching of the beam power supply. The reason is that the frequent switching of the beam power supply does not cause any problem. The demerit of this method is that the xenon is consumed even in the thrust-off period. This is, however, not a serious problem for the demonstration satellite.

The conditions of simulations are the same with those in the section 3.2.1. The thrust of the ion engine has been set to be $36 \mathrm{mN}$ in order to maintain the orbit at the solar maximum period. Since the launch of the demonstration satellite is thought to be near the solar maximum period, the maintenance control of two orbits (DLT=6h and $10 \mathrm{~h} 30 \mathrm{~m}$ ) were simulated for about one year at the solar maximum period. The maintained width of the altitude was set to be $\pm 0.5 \mathrm{~km}$. 


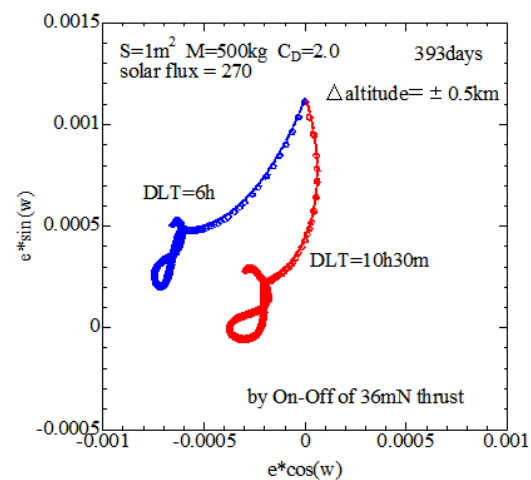

Fig.9. Changes of mean eccentricity

Fig.9 shows the two trajectories of the mean eccentricity vector for 393days. The "new eccentricity vector" moves with a period of one year primary due to the change of the declination of the sun. Incidentally, the simulation, where the on and off interval was not limited to a multiple of one orbital period, revealed large noises in the trajectory of the mean eccentricity vector.

Fig.10 shows the mean altitude change for 393days with the orbit of DLT $=10 \mathrm{~h} 30 \mathrm{~m}$. For DLT=6h, almost the same result was obtained. Since the simulation started from January 1st, it turns out that the air drag becomes large around the vernal and autumnal equinox.

From the studies described above, it was found that even the on-off thrust control can maintain the "new frozen orbit” accurately.

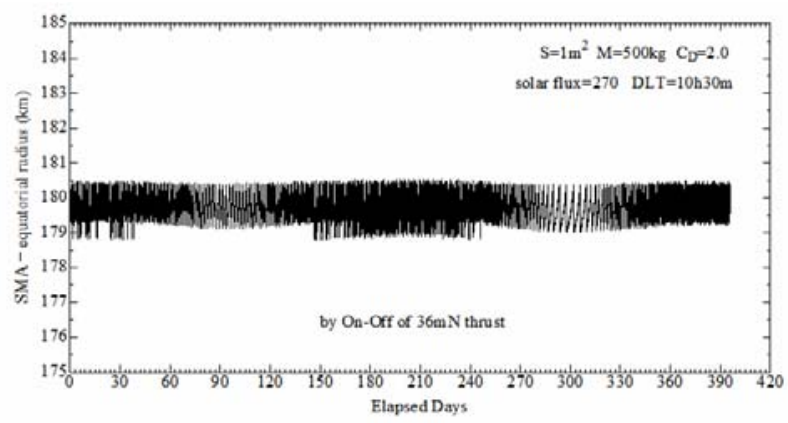

Fig.10. Altitude change $(\mathrm{DLT}=10 \mathrm{~h} 30 \mathrm{~m})$

\section{System Configurations}

We studied the satellite's system briefly. This satellite requires the solar battery with large area in order to supply electric power to the ion engine.

When the SAR or the LIDAR is installed, a sun synchronous orbit with the descending local time of 6:00 is preferable because of the minimum eclipse period.

When the optical camera is installed, a sun synchronous orbit with the descending local time of about 10:30 is preferable. It is difficult, however, for this orbit to secure the electric power, because this orbit has a long eclipse period and the period in which the sun incidences obliquely to the solar battery is long. Therefore, this orbit requires the solar battery with larger area.

\subsection{Example of system configuration with optical camera}

Fig.11 depicts an example of the external appearance of the satellite with an optical camera.

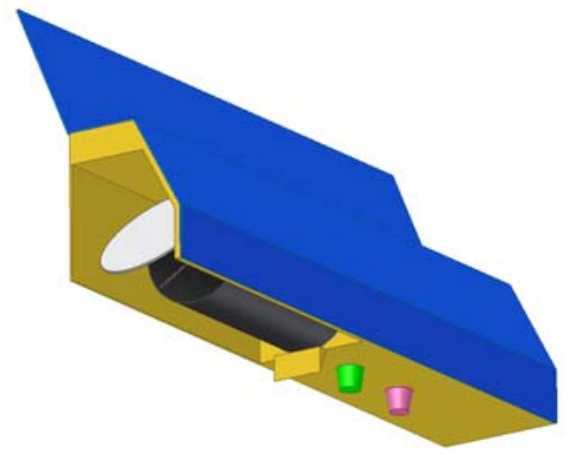

Fig.11. Example of optical camera satellite

Table 4-1 shows an example of system parameters for optical camera mission.

Table 4-1. System parameters for optical camera mission

\begin{tabular}{|l|r|l|}
\hline items & \multicolumn{1}{|l|}{ values } & remarks \\
\hline resolution & $30 \mathrm{~cm}$ & Nadir \\
\hline aperture & $50 \mathrm{~cm}$ & \\
\hline orbit & $10: 30$ & \\
\hline local time & $0.37 \mathrm{~m}^{2}$ & \\
\hline front proj. area & $13.2 \mathrm{mN}$ & average \\
\hline required thrust & $5.5 \mathrm{~m}^{2}$ & \\
\hline solar battery area & $1900 \mathrm{~W}$ & Max \\
\hline generated power & $500 \mathrm{~W}$ & non-obs* \\
\hline consumed power & $34 \mathrm{~kg}$ & for 2years \\
\hline fuel amount & $600 \mathrm{~kg}$ & \\
\hline satellite mass & & \\
\hline
\end{tabular}

*) period of no observation

\subsection{Example of system configuration with SAR}

Fig.12 depicts an example of the external appearance of the satellite with SAR mission.

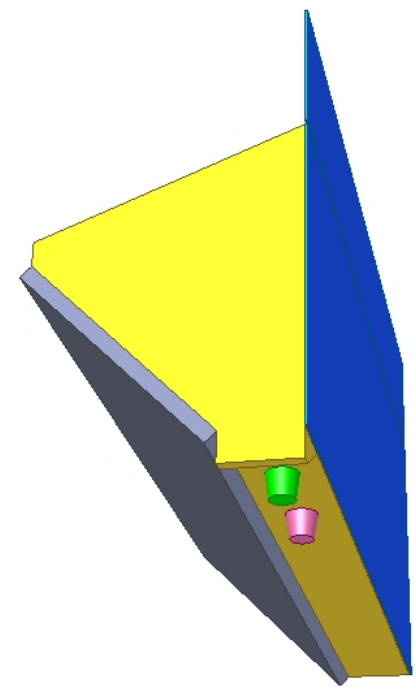

Fig.12. Example of SAR satellite 
Table 4-2 shows an example of system parameters for SAR mission.

Table 4-2. System parameters for SAR mission

\begin{tabular}{|c|c|c|}
\hline items & values & remarks \\
\hline resolution & $3.5 \mathrm{~m}$ & nadir \\
\hline frequency & L band & \\
\hline orbit & sun sync & \\
\hline local time & $6: 00$ & \\
\hline front proj. area & $0.86 \mathrm{~m}^{2}$ & \\
\hline required thrust & $30.6 \mathrm{mN}$ & average \\
\hline solar battery area & $5.0 \mathrm{~m}^{2}$ & \\
\hline generated power & $1700 \mathrm{~W}$ & $\max$ \\
\hline consumed power & $1000 \mathrm{~W}$ & non-obs \\
\hline fuel amount & $77 \mathrm{~kg}$ & for 2years \\
\hline satellite mass & $700 \mathrm{~kg}$ & \\
\hline
\end{tabular}

\section{Future Tasks}

Future tasks for the super low altitude satellite are described below.

\subsection{Performance confirmation of ion engine}

Especially for the satellite with accurate altitude maintenance requirements, the throttling of the thrust will be required.

There may be required to develop new ion engine, because the flexibility of the thrust and the durability of the engine, which have not been realized until now, are desirable to this satellite.

\subsection{Measures against deterioration by the atomic oxygen}

Since the atmosphere around the altitude of about $180 \mathrm{~km}$ contains a lot of atomic oxygen, it can deteriorate the materials of satellite and may cause some unexpected issues.

Since the atomic oxygen impinges the satellite mainly from the front side, the protection of the front side by the shield such as a bumper will be effective. The frequency of impingement from other directions should be studied.

Furthermore, we have to select the materials which are less influenced by the atomic oxygen. This might increase the cost of the satellite.

\subsection{Installation of long and slim satellite into the rocket}

The duration of the visible time from a ground station is very short for the super low altitude satellite. Therefore, the autonomous orbital maneuver based upon the onboard GPS receivers is inevitable.

Especially when troubles such as the breakdown of the ion engine happen, it will be necessary to raise the altitude of the satellite automatically.

\subsection{High speed data transmission}

For the optical camera mission, the data rate will be very high. Nevertheless, the visible time from a ground station is less than about 5 minutes.

To mitigate this problem, inter-satellite communication might be a solution, but the data transmission with the inter-satellite communication brings a different severe problem. Since the distance between satellites is long, the super low altitude satellite has to install a large high gain antenna which will raise the air drag. Therefore, both the satellite size and its cost will become large.

Considering those mentioned above, direct transmission with a high data rate antenna will be necessary.

5.5. Installation of long and slim satellite into the rocket

The super low altitude satellite must have a small front projection area. When the satellite is installed in a launch vehicle, the long and slim shape has to be considered to mount on it.

\section{Engineering Test Satellite}

We are planning to develop a engineering test satellite which will perform the tasks described in the previous chapter and demonstrate the superiority of orbiting at about $180 \mathrm{~km}$. The demonstration satellite will be launched as early as 2011, and become a precursor for operational super low altitude satellites.

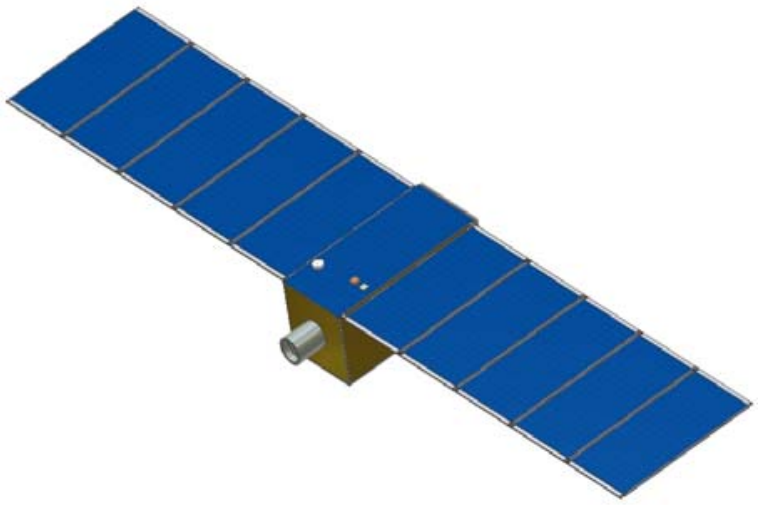

Fig.13. Example of Engineering test camera satellite

\section{References}

1) V.A.Chobotov, Editor, “Orbital Mechanics,” AIAA Education Series, 1991, pp.291-296.

Fig.12 depicts an example of the external appearance of the satellite with SAR mission. 